\title{
Towards an extended interoperability systemic approach for Dynamic Manufacturing Networks: role and assessment of PLM standards
}

\author{
Emna Moones ${ }^{1,3}$, Nicolas Figay ${ }^{2}$, Thomas Vosgien ${ }^{1}$, Lyes Kermad ${ }^{3}$, \\ François Stephan ${ }^{1}$, Abderrahman El Mhamedi ${ }^{3}$, El Mouloudi Dafaoui ${ }^{3}$
}

\begin{abstract}
This paper aims at illustrating some limitations of the systemic approach when willing to ensure the interoperability of PLM solutions within a Dynamic Manufacturing Network (DMN), based on e-Business PLM standards and their implementations, being industrial processes, methods, applications or Information \& Communication Technologies (ICT) solutions. Indeed, addressing interoperability challenges in such a complex digital business eco-system calls for a holistic approach based on the "system" paradigm. Setting this way, a part of our goal is to underline the limits and drawbacks of such an approach as interoperability brakes and to derive the issues that must be addressed in terms of research in order to remove them. This paper introduces a new approach in order to set up a test bed environment for PLM standards. The required and proposed approach considers a PLM standard not only as a technical solution, but above all as a strategic solution for which it is mandatory to support and enhance discussions between enterprise, product/system, processes, ICT architects and designers. The proposed approach - for analyzing and assessing the relevancy of PLM standards regarding their usage in specific business contexts - will be illustrated with a multi-layer modeling language. This language is used to model standards-based business collaboration scenarios and to model the test bed environment that will enable the execution/simulation of this scenario and the assessment of related standards implementations regarding the business needs of the scenario. The addressed case study is based on a data exchange scenario between a customer production order scheduler and a supplier production order executer using the ISA 95 standard. From this example, the interoperability issues related to DMN system of systems will be identified, for which accurate test based methods will be defined in future work.
\end{abstract}

Keywords: System of Systems, Interoperability, Dynamic Manufacturing Network, PLM standards, ISO STEP, ISA 95

\footnotetext{
${ }^{1}$ Technological Research Institute SystemX, Palaiseau, France

\{emna.moones, thomas.vosgien, francois.stephan\}eirt-systemx.fr

${ }^{2}$ Airbus Group Innovations, 12 rue Pasteur 92150 Suresnes, France

nicolas.figay@eads. net

${ }^{3}$ University Paris8, 140 rue Nouvelle France, 93100 Montreuil, France

\{1.kermad, a.elmhamedi, e.dafaoui\}@iut.univ-paris8.fr
} 


\section{$1 \quad$ Introduction}

\subsection{Industrial context}

In addition to System Engineering (SE), one trends for manufacturing industry is the application of Product Life cycle Management (PLM). In [1], CIMDATA defines PLM as strategic approach aiming to put in place appropriate processes and solutions for creation and sharing of Product Data and associated processes. PLM applies between enterprises involved in the different phases of the life cycle of the manufactured products and of its components. As PLM solutions relies today systematically on software-based solutions, Product data are digital and have to be interpreted at the same time by the different actors and by technical applications (e.g. Computer Aided Design/Manufacturing solutions) of the numerous enterprises and organizations concerned by the manufactured product. As a consequence, digital e-Business ecosystems are emerging, constituting Dynamic Manufacturing Networks (DMN) for which interoperability of technical applications is a major issue as defined in [2]. In DMN context, interoperability is the ability of the enterprises concerned by a manufactured product to enter the network by interconnecting their private processes, the applications and related technologies supporting these processes in order to ensure secured product and process data exchange and sharing.

In order to respond to digital collaboration needs, numerous industrial groups have been setting up PLM harmonization initiatives (e.g. EADS PHENIX ${ }^{4}$ ) for which importance of e-Business PLM standards were identified. In such a context, PLM standards are not technical solutions, but strategic solutions that have to be managed consistently by a community of interest (e.g. Aeronautic, Space \& Defense European and worldwide community). Importance of selecting and governing a relevant set of open e-Business PLM standards managed in configuration has been identified in different domains, in particular Aeronautic, Space \& Defense European community (c.f. ASD SSG [3]). Relying on standards [4] [5] and on models of reference for a community of reference [6] is the only way to achieve continuous and pragmatic interoperability at an acceptable cost. But some barriers remain for achieving such interoperability. In particular the ability for industry to effectively specify how software solution providers must implement standards in order to support their business collaborative processes and in order to facilitate application testing and deployment in industrial operational context.

In addition to the PLM approach and in order to deal with increasing complexity of economic environment, organizations and products, enterprises are also investing more and more in SE. According to INCOSE [7] (International Council of System Engineering), "SE is an interdisciplinary approach and means to enable the realization of successful systems. It focuses on defining customer needs and required functionality early in the development cycle, documenting requirements, and then proceeding with design synthesis and system validation while considering the complete problem. SE integrates all the disciplines and specialty groups into a team effort forming a structured development process that proceeds from concept to production to operation". SE community has been developing its own set of standards, such as ISO 15288

\footnotetext{
${ }^{4} \mathrm{http}: / /$ www.journeeduplm.fr/uploads/file/eads.pdf
} 
[8], system modeling languages such as SysML [9] or SESTEP application protocol (ISO10303-233) [10].

Some overlapping exists between PLM and SE. According to ISO 15288, the system of interest, i.e. the manufactured product, and the supporting systems, i.e. system for designing, producing, operating and supporting the product, are distinguished. For each of them, all of the phases of the lifecycle are to be considered in order to ensure adequacy between industrial processes and enterprises' capabilities. So stated, it seems that PLM is included inside SE. But the scope of application of PLM is larger than the one covered by SE processes and can be applied being SE processes independent. PLM is also more concerned by the information system and by the technical applications, while SE is more concerned by engineering methods and processes.

Finally, both SE and PLM are concerned by interoperability. While PLM is concerned by data exchange, sharing and long term archiving, SE is concerned not only by possible interaction between systems and by automated reconfiguration of system of systems (SOS) but also by enhancing communication and hence interoperability between multi-disciplinary design teams. Moreover, SE also focuses on the adaptation of the overall system in order to respond to the targeted objectives and on the way the different sub-systems of a SOS have to be aggregated dynamically and to interact easily.

\subsection{Research context and orientation of the proposal}

The research work presented in this paper is related to the research project "Standards Interoperability PLM" (SIP $\left.{ }^{5}\right)$ launched within the frame of the IRT-SystemX. As defined in [5], this project has three main objectives. First objective is the development of a generic approach and framework for specifying and testing implementation of PLM standards for multi-disciplines and multi-sectors collaboration. Second objective is the promotion of an experimental capability research for developing, assessing and implementing a configured set of relevant PLM standards which covers the whole phases of the life cycle of an industrial product. Third objective is the enhancement of the PLM Interoperability maturity of industry: for any stakeholder or actor of the domain, it is allowed accessing, assessing and contributing to the results of the project. The goal is to create a sustainable (i.e. which will continue to exist after the end of the project) open community which will be able to drive development by software product providers of accurate PLM solutions with validated specifications and ability to test them within a DMN.

Because PLM and SE are closely related, the project has to consider standards and practices of both PLM and SE communities. The project also develops a global interoperability approach that will extend SOSI approach taking into account virtualization aspects. Our goal in this paper is limited to point out an interoperability brake which complete the set of brakes defined in [11], and related to some limitation of system paradigm for DMN Interoperability.

The section 2 of the paper will describe the research foundation of the SIP approach. The section 3 will describe and analyze the limitations of systemic for addressing interoperability. The section 4 will illustrate the SIP approach and the previ-

\footnotetext{
${ }^{5}$ http://www.irt-systemx.fr/systemx-lance-le-projet-sip-standards-interoperabilite-plm/
} 
ously described limitations for a case study related to the ISA 95[12] standard, and to enterprise control integration. Conclusion will introduce perspectives and future work.

\section{SIP related work}

\subsection{Positioning according Interoperability state of the art}

The SIP approach is closely related to [11], in which the author proposes a federated framework for interoperability of technical enterprise applications. This framework first states what is an enterprise application and what is interoperability of enterprise applications. It then qualifies the "ideal" information system for networked collaborative product development. On the basis of past research projects and operational projects, the author also analyzes why PLM standards are not used, identifying set of interoperability brakes (i.e. what lead to non-interoperability) and interoperability enablers. Enablers include those defined in the ATHENA project [13], which considers that interoperability must be addressed at different layers - business, knowledge and ICT (Information and Communication Technologies) - with inter-related ontological models defined at each layer. In addition, a model driven approach is used in order to "project" the business logic (business objects, services and processes) on a service oriented execution platform including service bus, application servers and workflow engines.

Complementary proposed enablers are the systematic usage of open standards, the need for preparing and constructing operational interoperability as defined by SOSI. Another important identified enabler is the establishment of a community of interest to build its maturity through the governance of a consistent set of standards covering their needs. The brakes can be considered as practices adopted by enterprises which are going against interoperability. An example is the management by project. As a project has restricted duration and scope, long term and global strategic approach at enterprise scale are usually not considered. As a consequence, using a neutral standard for interchange is considered at the project scale as an important extra cost, and is often not considered as a first priority. Needs for management, evolution and consistency of the whole enterprise information system are not considered.

Alternating research projects and operational projects with continuous update of enablers and brakes is another principle of the framework. Doing so, the framework has been completed through Crescendo [14] project for integration of an enterprise portal as part of the execution platform, in particular in order to deal with controlled access to resources of the enterprises. The brakes addressed here is security, which is a stopper when not achieved. Standards for simulation were considered such as ISO10303-209 [15] and CGNS [16]). The IMAGINE project ${ }^{6}$ has been addressing dynamic allocation of actual qualified resources to a process within a DMN. Concerned applicative resources might implement PLM standards in order to support seamless information flow all along cross-organizational collaborative project. In addition, usage of virtualization servers has been adopted in order to facilitate deployment, set up, and simulation of an actual DMN over the public or private clouds. Finally, ArchiMate has been adopted as the open standard to be considered for enter-

\footnotetext{
${ }^{6} \mathrm{http}: / /$ www.imagine-futurefactory.eu/index.dlg/
} 
prise modeling promoted in ATHENA. ISA95 and ISO15288 were assessed and combined. The brake addressed by IMAGINE is the lack of methodology for qualification of a set of applications involved in a cross organizational collaborative process.

\subsection{Positioning according test beds state of the art}

SIP was built on top of the results of these successive projects, in order to address brakes related to missing methodology for producing use cases, business scenarios, test data sets and test procedure, positively impacting implementation costs for making solution providers implement the standards. Referring to existing test beds such as NIST QOD and CVTS ${ }^{7}$, Korean B2B interoperability test bed, Global eBusiness Interoperability test beds (GITB) or Agile Test Framework (ATF), it appears that none of them is addressing the need to consider implementation of standards by the engineering processes first before to specify implementation of interfaces within commercial solutions.

The innovative aspect of the SIP project concerning test beds is also the ability to consider several standards in a holistic way, with combined usage of Business standards (e.g. ISO 15288 technical processes), applicative standards (e.g. application data interchange protocols such as ISA 95 or ISO STEP) and ICT standards for data exchange (e.g. XML [17]), distributed services (e.g. WSDL [18]) or process choreography (e.g. XPDL [19]). All these standards are mapped within enterprise models formalized with ArchiMate, allowing enterprise, business process, product, information system and ICT architects to establish PLM interoperability through industrialization of standards.

\subsection{SIP and System Engineering}

SIP is closely related to SE by several aspects. First the SE process framework defined by ISO 15288 is used for contextualization of PLM standards, but also for making a clear distinction between the system of interest (e.g. an aircraft) and supporting systems (i.e. system for designing, system for producing, system for operating or system for supporting). Then the SysML standard is one of the PLM standards considered for the support of some of the technical processes related to requirement engineering, design and simulation. In addition, usage of model driven approach for referential component relies on the Unified Model Language, which support both object and component paradigms. An object groups data and methods for systems which have to interact through exchange of messages. It considers what is internal to objects and what is external. Internal part can be accessed through public interfaces. Object classes are used for categorization of objects, and support inheritance mechanism in order to ensure reusability. Such mechanism brings an important drawback, due to the complexity of inheritance trees and usage of specialization/generalization for combining business, applicative and technical objects. It led to the failure of standards such as PDM Enablers, too difficult to implement as it required mastering business and ICT specifications. The "component" paradigm provides the concept of container: a container is provided by an application server and allows deploying business objects.

\footnotetext{
${ }^{7}$ http://www.nist.gov/el/msid/qod_standalone_release.cfm/
} 
ICT services provided by an application server can be applied to the business through the containers, according to policies applied to these containers. Doing so, business and ICT aspects are decoupled, allowing separation of concern. Business logic can be deployed as engineering artefacts on top of execution platform. It is so possible to execute business logic.

Finally, when considering DMNs, it is required to interconnect legacy applications supporting organization which have to collaborate dynamically, with continuous evolution of organizations, processes and ICT leading to dynamic reconfiguration if willing to ensure continuous interoperability. It seems that DMNs can be considered as SOS, and consequently it should be possible to use methods and tools defined by the SOS community. It was done with SOSI principles, which define operational interoperability which has to be prepared by mean of governance (c.f. ASD SSG) and constructed by mean of architectural patterns. But some issues exist when using the "system" paradigm. It can be analyzed comparing our global interoperability approach (taking into account virtualization aspects) used in SIP and other systemic approaches like SOSI.

\subsection{Architecture and principles of SIP test bed}

The SIP test bed (c.f. Figure 1), includes first an execution platform combining standardized collaborative portal, workflow engine and enterprise service bus. On this execution platform, testing and standard based PLM services are deployed while a shared repository of use cases, test scenarios and test data set. Infrastructure and processes of the SIP test bed make possible controlled access to the services and the repository, which can be public or restricted to a given community or enterprise.

The SIP methodology allows generating referential implementations of applicative components from models (based on MDA [20]) that will simulate the different kind of applications (type A or B in the figure) that will be interconnected for supporting the collaboration.

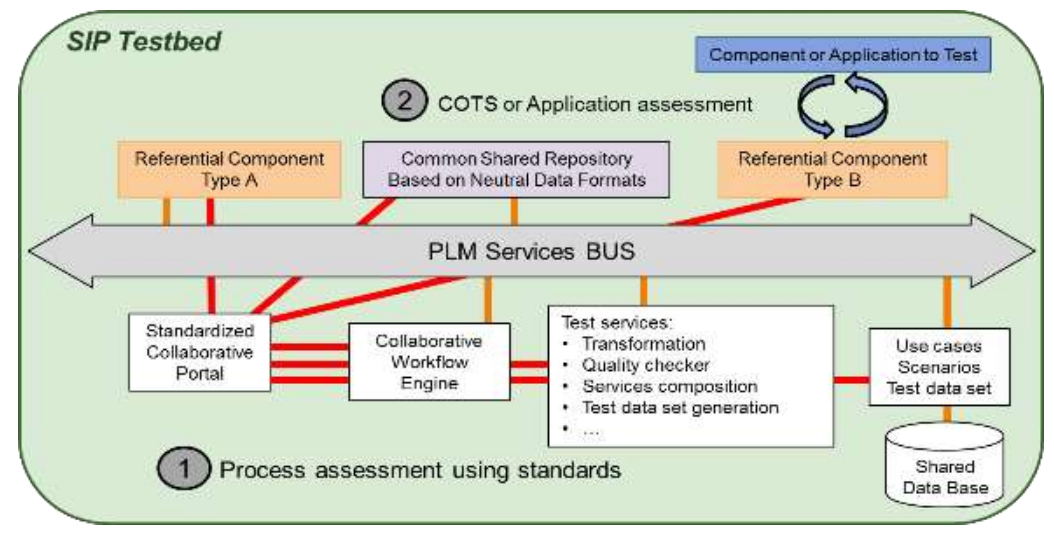

Fig. 1. SIP test bed architecture and principles 
Once these components generated, deployed and interconnected, it will then be possible to simulate standards-based collaboration and then to assess as well the used standards implementations (are they covering business needs?) than the cross organizational collaborative processes of a given digital business ecosystem such as Technical data package exchanges, Change and Configuration process management, etc. Once validated by the mean of the test bed, enterprises will then be able to precisely specify to software solution providers and to integrators what is needed in order to be able to interconnect actual applications and their interfaces. When actual applications and their interfaces ready, it will then be possible to test them reusing the test bed: referential components will be unplugged, and replaced by the actual application. By playing the same test scenarios and reusing the same test data, it will be possible to assess and qualify actual applicative components, performing first unitary tests (one component alone) and integration tests for a whole end to end process involving at the same time different organizations, different applications and potentially a set of different PLM standards.

SIP will not perform all the work, but invite partners and communities to apply SIP methodology and to use and enrich the SIP platform with new open components in order to build maturity of the industry concerning PLM interoperability, being for design, production or integrated logistic support.

\section{Systemic and its limitations for a global interoperable PLM approach}

The theory of systems was founded by Ludwig von Bertalanffy, William Ross Ashby and others between 1940 and 1970. It evolved as the study of the complexity, with a particular focus on dynamic and evolutionary systems. Systemic analysis is an interdisciplinary field related to the study of complex objects which can be understood with classical approaches (e.g. Cartesian method), such as living being or electronic systems for temperature regulation. In order to face such a problem, it is required to adopt a holistic approach, focusing more on the exchange and interactions (interaction, retroaction, regulation) between the different parts of a system than on the analysis of each part, and considering the objective of the system (teleology). Systemic approach is applied to numerous domains: biology, architecture, etc. It relies on visual modeling, descriptive or formal, executable or not. With executable models, it is possible to use simulation. As stated by AFSCET [21], the problem of boundaries is a key when willing to deal with what is internal and external and to be able to define the interactions between the systems.

Looking at the complexity of a DMN, it seems that systemic approach could be appropriate for addressing PLM interoperability. As for systemic approach, the SIP interoperability approach is holistic, and considers different systems: the system of interest (i.e. the product), the supporting systems, the information system, the enterprises, the digital business eco-systems, etc. Nevertheless we identified some difficulties concerning usage of systemic approach in the PLM interoperability context, when willing to define boundaries for a system. The origin of this difficulty is the virtualization. 
Virtualization refers to the act of creating a virtual (rather than actual) version of something. This has been used since a long time in computer science, with as an example usage of logical disk names in order to be able to change the actual used physical disk in a transparent way without impacting the users. Virtualization has many other usage and applications to be considered in SIP. Enterprise portals are software systems which aim to give users access to numerous applications through an integrated interface, hiding the complexity of the underlying actual architecture of solutions realizing these applications. It is true in particular for PLM hubs used by a digital business eco-system. In order to easily deal with a simulation platform, SIP is making intensive usage of virtualization servers in order to reduce time of deployment and replication of a whole collaborative network. Cloud computing and Grid computing are making extensive use of virtualization. Finally, enterprise modeling and associated standards are interconnecting the enterprise and the actual ICT system using business layer, applicative layer and ICT layer. The ICT layer is constituted of concrete devices and software systems used in order to realize an application. The applicative layer is purely logical, and makes the interface between ICT technologies and the business. ICT devices and software systems are concrete actual systems that are owned by an organization, which have physical location and which are physically operated through accurate processes by organizations. Owner of the ICT capabilities, ICT capabilities and operators of the ICT capabilities can as well be inside or outside the enterprise using the application.

As a consequence, as soon as virtualization is used, it is not possible to preserve boundaries of a system between Business and ICT layers. When interactions exist between two organizations, there are not necessarily interactions between two software systems installed on different machines. Conversely, one organization can access one application without knowing it implies interaction between numerous software systems distributed on different machines and eventually hosted within numerous organizations. Considering grid computing which allocate dynamically available actual resources to an application, it is impossible to predict what will be the actual used resources and where they will be located.

\section{$4 \quad$ Illustration through ISA95 case study}

\subsection{ISA 95 standard for Enterprise control integration}

ISA-95 is an international multi-part set of standards that defines interfaces between enterprise activities and control activities. Developed for global manufacturers, it applies in all industries and in all sorts of processes, like batch processes, continuous and repetitive processes. Four functional levels are defined by ISA 95 standard. Levels 0,1 and 2 are the levels of process control. Their objective is the control of equipment, in order to execute production processes that end in one or more products. Level 3 could be called the level of MES (manufacturing execution system) activities, it consists of several activities that must be executed to prepare, monitor and complete the production process that is executed at level 0,1 and 2. The highest level (level 4) could be called the level of enterprise, including ERP (Enterprise Resource Planning) systems and PDM Systems. At this level financial and logistic activities are executed 
in order to produce the product configuration ordered by the client. ISA 95 focus on the Production system, which is a supporting system according ISO 15288. The system of interest (ISO 15288) is the product, which is the output of production activities (ISA 95). The ways for describing Product data are very different between Production departments (ISA 95, B2MML), design offices (ISO STEP AP242) or customer support department (ISO STEP ISO AP239), as the purpose and the goal of their activities are not the same. A PLM approach should address consistent usage of this set of standards.

\subsection{Modelling and simulation of a DMN collaboration process}

On Figure 2, the different applications are realized through simulators hosted on the test bed (referential components) and virtualized on the cloud. As a consequence, the physical realization of the applications is out of the enterprise systems boundaries. When real application is ready to be tested, the referential component is unplugged and replaced by the real application. It is fully transparent at business layer where used solutions realizing the applications are hosted.

The Figure $3^{8}$ is an illustration of captured processes covered by ISA 95, underlying information system and ICT layer, including the SIP test bed infrastructure.

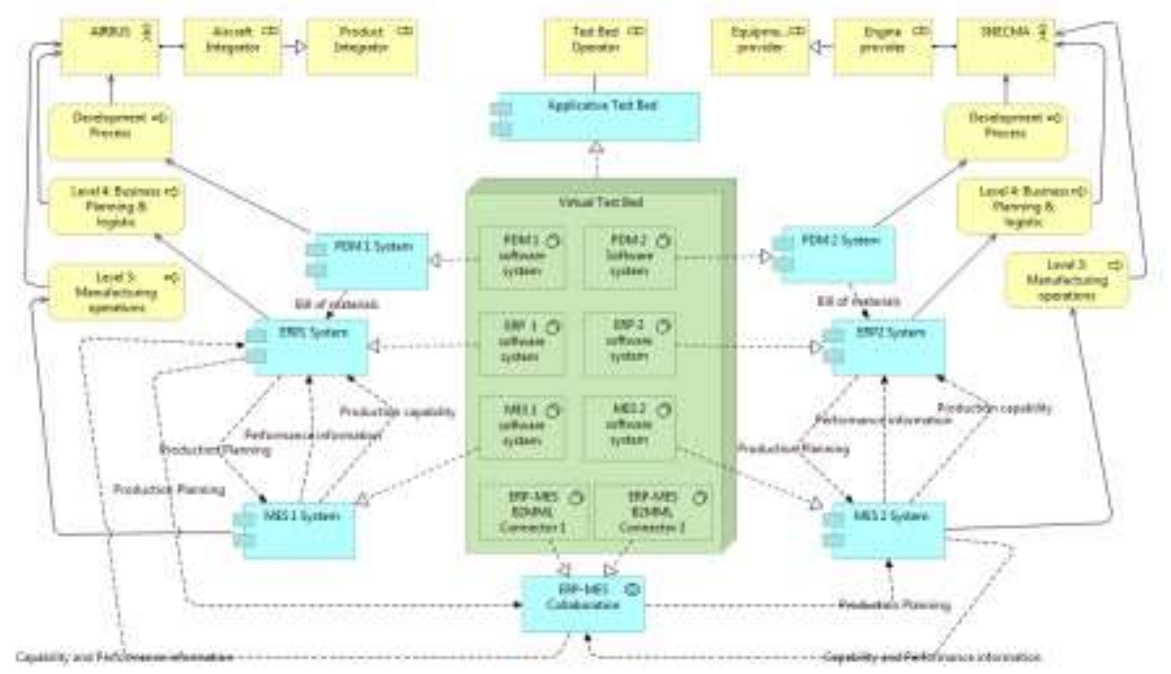

Fig. 2. ArchiMate view of SIP test bed for ERP/MES/PLM solutions

The upper part is the collaboration scenario model, which involves a "customer order scheduler" and a "supplier order executer" (grey). The order scheduler wants to transmit to its supplier a production schedule as a set of production requests (or orders) with associated required manufacturing bill of materials respectively from its ERP and PDM systems. The supplier might be able to integrate all these information into its MES system in order to define its detailed production planning. This scenario

\footnotetext{
${ }^{8} \mathrm{http}: / /$ www.eads-iw.net/image/image_gallery?img_id=162879\&t=1404673922104/
} 
is modeled on the three layers. The business layer (yellow) specifies the sequence of business activities and related business objects. The applicative layer (blue) specifies the applicative components supporting these activities and the data objects realizing the business objects. The technological layer (green) specifies the actual software systems and the data files respectively realizing the applicative components and data objects.

The lower part represents the test bed model; i.e. the applicative (blue boxes on the right side) and technological infrastructure (green boxes on the left side) for simulating the collaboration scenario and assessing exchange sequences and related ISA95 implementations. In the technological layer (the virtualization platform), we modeled and distinguished the virtual physical cluster specifying the physical and "real" infrastructure nodes describing a set of virtual machines and the virtual test bed composed of these latter. These virtual machines host the simulated applications/implementations, and the physical storage devices host the generated or consumed test data sets but also enabled the workflow models of the test scenarios. Figure 3 highlights not only the complexity of modelling such a collaboration scenario on the three layers, but also the difficulty to define system boundaries that can be preserved between the business, applicative and ICT layers of a complex DMN model. 


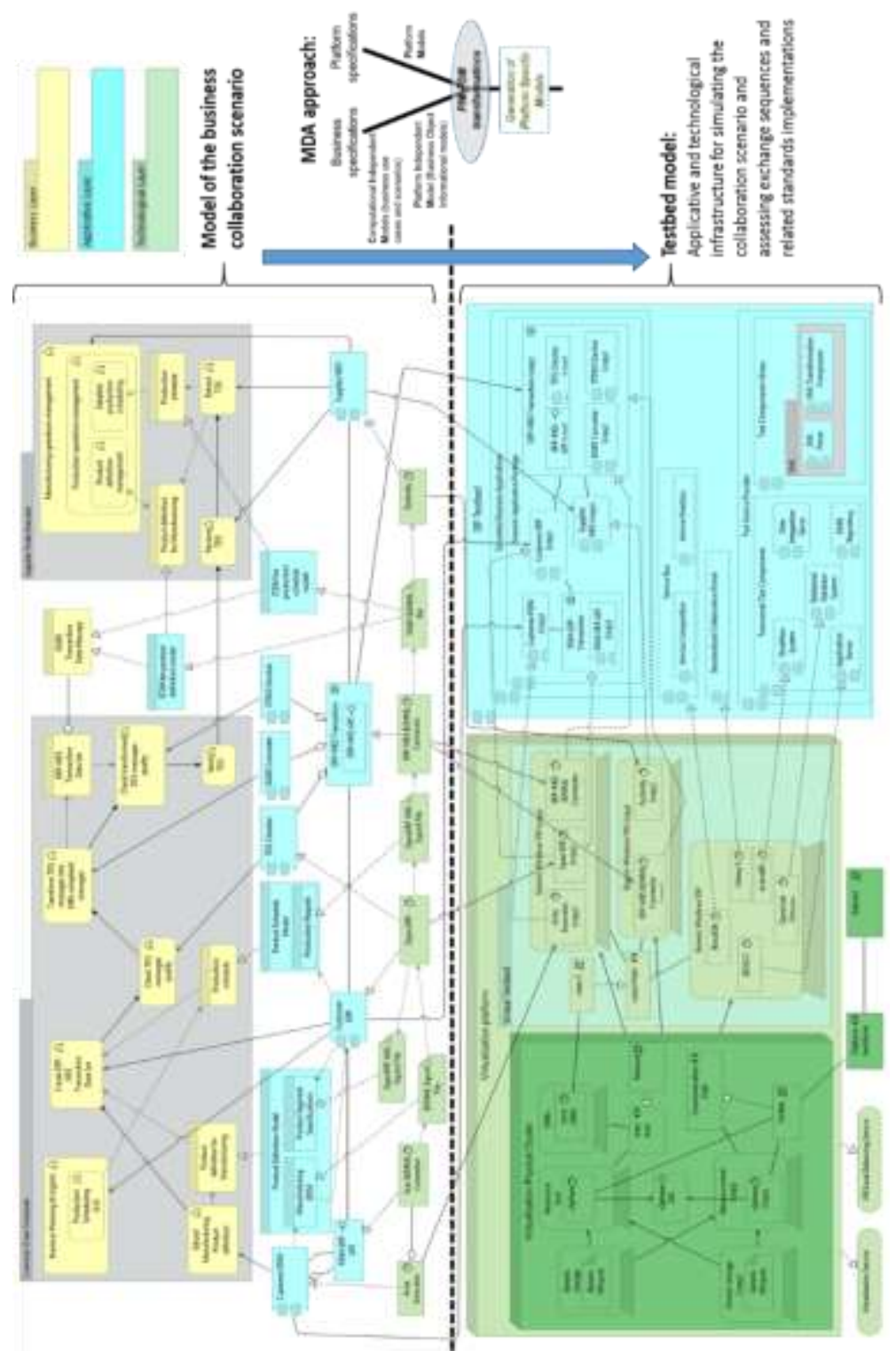

Fig. 3. ArchiMate view of ISA95 business case on top of SIP Test bed 


\section{Conclusion and way forward}

In this paper, we have illustrated some limitations of the systemic approach when willing to ensure the interoperability of PLM solutions within a DMN. It was done within the context of our research activities related to PLM interoperability based on standards. We aims to propose a methodology, which was just introduced in this paper, for dealing with DMN, based on a holistic approach derived from the federated interoperability framework, and addressing the interoperability brake related to missing adapted approach for use cases and test scenarios. The methodology, relying on a test bed allowing execution and simulation of DMN models, and the approaches developed for system of systems, are very similar: high complexity, iterative usage of modeling and simulation. We also reuse SOSI concepts, for preparing and constructing operational interoperability, and we rely on System Engineering process framework for contextualization of PLM standards regarding System Engineering process standards. But due to virtualization, it is not possible to define system boundaries that can be preserved between the business, applicative and ICT layers of our DMN models. We have illustrated some limitations of the systemic approach and system paradigm by modeling a business case related to the usage of the ISA 95 standard, for inclusion of the production function in an interoperable PLM approach. The methodology we are developing will address such limitations, but also other interoperability brakes we identified and that will be described in future papers. We recommend also considering the lifecycle impact of design/support tools for software components in the system of interest and what advantages could the use of web based interoperability technologies provide. Our approach will apply to manufacturing standards for production systems, but also to design systems (Computer Aided Design, Configuration Management and Simulation) and integrated logistics systems.

\section{Acknowledgement}

This research work has been carried out under the leadership of the Technological Research Institute SystemX, and therefore granted with public funds within the scope of the French Program "Investissements d'avenir".

\section{References}

1. CIMDATA: "All about PLM", https://www.cimdata.com/en/resources/about-plm, (2014).

2. Figay, N., Tchoffa, D., Ghodous, P., Exposito, E., El Mhamedi, A.: Dynamic Manufacturing Network, PLM Hub and Business Standards Testbed. In Enterprise Interoperability VI (pp. 453-463). Springer International Publishing (2014).

3. ASD SSG: ePLM Interoperability, http://www.asd-ssg.org/ (2014).

4. J, Lianga., J,J, Shaha., R, D’Souzaa., S,D, Urbanb., K, Ayyaswamyb., E, Harterc., T, Bluhmc.: Synthesis of consolidated data schema for engineering analysis from multiple STEP application protocols Computer-Aided Design 31, (1999).

5. X, F, Zha., H, Du., A.: PDES/STEP-based model and system for concurrent integrated design and assembly planning Computer-Aided Design 34, (2002). 
6. ATHENA: "Interoperability Framework v2.0", http://www.nehta.gov.au/implementationresources/ehealth-foundations/EP-1144-2007/ (2007).

7. INCOSE: International Council on Systems Engineering, http://www.incose.org/ (2014).

8. International Organization for Standardization: ISO 15288:2008 - Systems and software engineering - System life cycle processes, (2008).

9. Object Management Group: SysML Version 1.3, http://www.omg.org/spec/SysML/1.3/ (2012).

10. International Organization for Standardization: ISO 10303-233:2012 - Industrial automation systems and integration - Product data representation and exchange - Part 233: Application protocol: Systems engineering, (2012).

11. Figay, N.: Interoperability of technical enterprise applications, Doctoral Thesis, University Lyon 1, (2009).

12. ISA-95: the international standard for the integration of enterprise and control systems, http://www.isa-95.com/ (2014).

13. Athena Interoperability Framework (AIF), http://athena.modelbased.net/ (2010).

14. CRESCENDO: Collaborative and Robust Engineering using Simulation Capability Enabling Next Design Optimization, http://www.crescendo-fp7.eu/ (2014).

15. International Organization for Standardization: ISO 10303-209:2001 - Industrial automation systems and integration - Product data representation and exchange - Part 209: Application protocol: Composite and metallic structural analysis and related design (2001).

16. CGNS, NASA, CFD: General Notation System, Version 3.2.1 http://www.grc.nasa.gov/WWW/cgns/CGNS docs current/ (2014).

17. W3C: Extensible Markup Language (XML), Version 1.0, http://www.w3.org/TR/xml/ (2013).

18. W3C: Web Service Definition Language (WSDL), Version 1.1, http://www.w3.org/TR/wsdl/ (2001).

19. WFMC: XML Process Definition Language (XPDL), http://www.xpdl.org/ (2014).

20. OMG: "MDA - The Architecture of Choice for a Changing World", http://www.omg.org/mda/ (2014).

21. Gérard Donnadieu., Daniel Durand., Danièle Neel., Emmanuel Nunez., Lionel SaintPaul.: L'approche systémique: de quoi s'agit-il, Synthèse des travaux du Groupe AFSCET «Diffusion de la pensée systémique », http://www.afscet.asso.fr/SystemicApproach.pdf, (2010). 